\title{
THE PERIODIC SOLUTIONS OF THE SECOND ORDER NONLINEAR DIFFERENCE EQUATION
}

\author{
Ryszard Musielak and Jerzy Popenda
}

Abstract

Periodic and asymptotically periodic solutions of the nonlinear equation $\triangle^{2} x_{n}+a_{n} f\left(x_{n}\right)=0, n \in N$, are studied.

In several recent papers $([2],[3])$ the periodicity of solutions of linear difference equations have been investigated. In this paper we examine the periodic solutions of the nonlinear equation

$$
\triangle^{2} x_{n}+a_{n} f\left(x_{n}\right)=0, n \in \mathrm{N},
$$

where $\mathbf{N}=\{0,1,2, \ldots\}, \mathbf{R}$ is the set of real numbers, $f: \mathbf{R} \longrightarrow \mathbf{R}$ and $a, x$ : $\mathbf{N} \rightarrow \mathbf{R}$ are sequences of real numbers.

Throughout the paper we use the following notations. By $\overline{0, t}$ we denote the set of integers $\{0,1,2, \ldots, t\}$. For the function $y: \mathbf{N} \rightarrow \mathbf{R}$ the forward difference operator $\triangle^{k}$ is defined

$$
\Delta y_{n}=y_{n+1}-y_{n}, \Delta^{k} y_{n}=\Delta\left(\Delta^{k-1} y_{n}\right) \text { for } k>1 .
$$

Definition 1. The function $y$ will be called $t$-periodic if $y_{n+t}=y_{n}$ for all $n \in N$. (Furthermore we suppose that no $t_{1}$ exists, $0<t_{1}<t$ such that $y_{n+t_{1}}=y_{n}$ for all $n \in \mathbf{N}$ and that $t>1$ ).

Definition 2. The function $y$ will be called asymptotically t-periodic $(t>1)$ if

$$
y=u+v,
$$

where $u$ is a t-periodic function and $\lim _{n \rightarrow \infty} v_{n}=0$.

Definition 3. We say that the equation $(E)$ has a $p_{t}-$ constant if there exists a constant $p \in \mathbf{R}$, such that the equation

$$
\triangle^{2} x_{n}+a_{n} f\left(x_{n}\right)=p
$$


has at-periodic solution.

We say that the equation $(E)$ possesses a $p_{i}^{\infty}$-constant if there exists a constant $p \in R$ such that $\left(E_{1}\right)$ has an asymptotically $t$-periodic solution.

Definition 4. The equation $(E)$ is said to have a $p_{t}$-function ( $\left.p_{i}^{\infty}-f u n c t i o n\right)$ if there exists a t-periodic function $p: \mathbb{N} \longrightarrow \mathbb{R}$ such that the equation

$$
\triangle^{2} x_{n}+a_{n} f\left(x_{n}\right)=p_{n}
$$

has a $t$-periodic (asymptotically $t$-periodic) solution.

Remark 1. Note that if $(E)$ has a $p_{t}$-constant (function) then $(E)$ has a $p_{t}^{\infty}$-constant (function) and if $(E)$ has not a $p_{t}^{\infty}$-constant (function) then it has no $p_{t}$-constant (function).

Theorem 1. Let $f: R \longrightarrow R$ be continuous on $R$ and $\lim _{n \rightarrow \infty} a_{n}=0$. Then the equation $(E)$ has not a $p_{t}^{\infty}$-constant for any $t>1$.

Proof: We show the proof for simplicity in the case $t=2$. Similar reasoning can be made for $t>2$.

Suppose that there exists a $p_{t}^{\infty}$-constant $q$ such that the equation

$$
\triangle^{2} x_{n}+a_{n} f\left(x_{n}\right)=q
$$

has one asymptotically 2-periodic solution $x$.

Let $x_{2 n} \longrightarrow C_{1}, x_{2 n+1} \longrightarrow C_{2}$ as $n \longrightarrow \infty, C_{1} \neq C_{2}$. Hence

$$
\begin{gathered}
\triangle^{2} x_{2 n} \longrightarrow 2 C_{1}-2 C_{2} \\
\triangle^{2} x_{2 n+1} \longrightarrow 2 C_{2}-2 C_{1} .
\end{gathered}
$$

As result of the assumption we obtain

$$
\begin{aligned}
& 2 C_{1}-2 C_{2}=q \\
& 2 C_{2}-2 C_{1}=q .
\end{aligned}
$$

The above system has a solution if and only if $q=0$, but in this case we obtain $C_{1}=C_{2}$, which is a contradiction.

Theorem 2. Let $f \neq 0$ on R. If the equation $(E)$ possesses a $p_{t}$-constant then $a$ is a $t$-periodic function.

Proof: Let $x$ be a $t$-periodic solution of $\left(E_{3}\right)$. Then $\triangle^{2} x$ is $t$-periodic. By virtue of the assumption $f \neq 0$ and we get

$$
\frac{\triangle^{2} x_{n}-q}{f\left(x_{n}\right)}=-a_{n}
$$


The left hand side of the above equality is a $t$-periodic function so the right hand side must also be $t$-periodic.

Remark 2. We can prove analogously that if $f \neq 0$ on $\mathrm{R}$, then $t$-periodicity of $a$ is the necessary condition for the existence of a $p_{t}$-function $q$ for the equation $(E)$. However in this case we do not require for $t$ to be the basic period. Eventually $a$ can be a constant function. It is easy to see that if $f\left(C_{1}\right)=0$ then the equation $(E)$ has $p_{1}$-constant $q=0$. Then a $t$-periodic solution takes the form $x \equiv C_{1}$.

By $i_{R}$ we denote the identy function on $R$.

Theorem 3. Let $a: N \longrightarrow \mathbf{R}$, let $f$ be a continuous function on $\mathbb{R}, f \neq 0$ such that the functions

$$
i_{R}+a_{n} f: \mathbf{R} \longrightarrow \mathbf{R}
$$

are surjections for every $n \in \mathrm{N}$. If

$$
\sum_{j=1}^{\infty} j\left|a_{j}\right|<\infty
$$

then the equation $(E)$ has a $p_{2}^{\infty}$-function for arbitrary $t \geq 1$.

Proof: Choose $t \geq 1$. By assumption there exist constants $C_{r}, r=1,2, \cdots, t$, $C_{i} \neq C_{j}, i \neq j$, such that

$$
f\left(C_{r}\right) \neq 0
$$

The case

$$
f\left(C_{r}\right)>0, r=1,2, \cdots, t
$$

will be considered. The proof for the other cases $f\left(C_{i}\right)>0, f\left(C_{j}\right)<0$ is similar.

By virtue of the continuity of the function $f$ there exist intervals

$$
I_{r}=\left[C_{r+1}-\delta, C_{r+1}+\delta\right], r=0,1, \cdots, t-1
$$

such that

$$
f(u)>0 \text { for } u \in I_{r}, r=0,1, \cdots, t-1 .
$$

From (2) it follows that

$$
\lim _{n \rightarrow \infty} \sum_{j=n}^{\infty} j\left|a_{j}\right|=0
$$


Let us denote

$$
D=\max _{0 \leq \tau \leq t-t}\left(\max _{u \in t,} f(u)\right)
$$

and

$$
n_{1}=\min \left\{n \in \mathbb{N}:: n=t k+t-1, D \sum_{j=n}^{\infty} j\left|a_{j}\right| \leq \delta\right\} .
$$

In the space $l^{\infty}$ of bounded sequences with the norm

$$
\|x\|=s u p_{i \geq 0}\left|x_{i}\right|
$$

we define the set $T$ in the following way:

$$
x=\left\{x_{i}\right\}_{i=0}^{\infty} \in T
$$

if

$$
\begin{gathered}
x_{r}=x_{t+r}=x_{2 t+r}=\cdots=x_{n_{1}-t+r+1}=C_{r+1}, x_{t k+r} \in I_{t k+r}:= \\
=\left[C_{r+1}-D \sum_{j=t k+r}^{\infty} j\left|a_{j}\right| ; C_{r+1}+D \sum_{j=t k+r}^{\infty} j\left|a_{j}\right|\right], \\
r=0,1, \cdots, t-1: k \in \mathbb{N} ; k>\frac{1}{t}\left(n_{1}+1-t\right) .
\end{gathered}
$$

The set $T$ is closed, convex and bounded. Furthermore, by diam $S$ we mean

$$
\operatorname{diam} S=\operatorname{stp}\{\|x-y\| ; x \in S ; y \in S\} .
$$

So

$$
\operatorname{diam} I_{t k+\tau} \longrightarrow 0 \quad \text { as } \quad k \longrightarrow \infty
$$

It is easy to find a finite $\epsilon-n$ et for every $\epsilon>0$. Therefore by Hausdorff's Theorem the set $T$ is compact. Let us define an operator $A$ for $x \in T$ as follows:

$$
A x=y=\left\{y_{i}\right\}_{i=0}^{\infty}
$$

where

$$
\begin{gathered}
y_{r}=y_{t+r}=\cdots=y_{n_{1}+r+1-t}=C_{r+1} ; r=0,1, \cdots, t-1 \\
y_{t k+1}=C_{r+1}-\sum_{j=t k+r}^{\infty}(j+1-t k-r) a_{j} f\left(x_{j}\right)
\end{gathered}
$$

for $k \in \mathbb{N}, k>\frac{1}{t}\left(n_{1}+1-t\right), r=0,1, \cdots, t-1$.

Let us observe that

$$
I_{t k+r} \subset I_{r}, r=0,1, \cdots, t-1, k>\frac{1}{t}\left(n_{ \pm}+1-t\right) .
$$


Hence

$$
\begin{gathered}
\left|\sum_{j=i k+r}^{\infty}(j+1-t k-r) a_{j} f\left(x_{j}\right)\right| \leq \\
\leq \sum_{j=i k++}^{\infty} j\left|a_{j}\right|\left|f\left(x_{j}\right)\right| \leq D \sum_{j=i k+r}^{\infty} j\left|a_{j}\right| .
\end{gathered}
$$

Therefore $y_{t k+r} \in I_{i k+r}, r=0,1, \cdots, t-1, k \in N, k>\left(n_{1}+1-t\right) / t$ and this means that $A: T \longrightarrow T$. Let us take an arbitrary sequence $\left\{x^{m}\right\}_{m=1}^{\infty}$ of elements of $T$ convergent to some $x^{0} \in T$ i.e.

$$
\left\|x^{m}-x^{0}\right\| \longrightarrow 0
$$

Hence we have

$$
\sup _{n \geq 0}\left|x_{n}^{m}-x_{n}^{0}\right| \longrightarrow 0
$$

as $m \longrightarrow \infty$. Let $\epsilon_{1}$ be an arbitrarily taken positive real number. By the uniform continuity of $f$ on the sets $I_{r}$ we have

$$
\left|u_{1}-u_{2}\right|<\delta \text { implies }\left|f\left(u_{1}\right)-f\left(u_{2}\right)\right|<\epsilon_{1} \text {. }
$$

From (10) it follows that

$$
\sup _{n \geq 0}\left|x_{n}^{m}-x_{n}^{0}\right|<\delta
$$

for $m \geq M(\delta)$. Let $y^{m}=A x^{m}, m \in \mathbb{N} ;$ then

$$
\begin{gathered}
\left\|A x^{m}-A x^{0}\right\|= \\
=\sup _{n>n_{1}} \mid \sum_{j=n}^{\infty}(j+1-n) a_{j} f\left(x_{j}^{m}\right)-\sum_{j=n}^{\infty}(j+1-n) a_{j} f\left(x_{j}^{0}\right) \| .
\end{gathered}
$$

By (9) the series

$$
\sum_{j=n}^{\infty}(j+1-n) a_{j} f\left(x_{j}^{m}\right), m \in N
$$

are absolutely convergent. Hence, by (11) and (12)

$$
\left\|A x^{m}-A x^{0}\right\| \leq \epsilon_{1} \sum_{j=n_{1}}^{\infty} j\left|a_{j}\right|
$$

so that the operator $A$ is continuous on $T$. By Shauder's Theorem there exists $z \in T$ such that $z=A z$. By definition of $A$ this element $z=\left\{z_{i}\right\}_{i=0}^{\infty}$ satifies

$$
z_{r}=z_{t+r}=\cdots=z_{n_{2}+r+1-t}=c_{r+1}
$$




$$
\begin{gathered}
z_{t k+r}=C_{r+1}-\sum_{j=t k+r}^{\infty}(j+1-t k-r) a_{j} f\left(z_{j}\right) \\
k>\frac{1}{t}\left(n_{1}+1-t\right), r=0,1, \cdots, t-1 .
\end{gathered}
$$

Applying the operator $\triangle$ to $z$ we obtain

$$
\begin{gathered}
\Delta z_{t k+r}=z_{t k+r+1}-z_{t k+r}= \\
=C_{r+2}-C_{r+1}-\sum_{j=t k+++1}^{\infty}(j+t k-r) a_{j} f\left(z_{j}\right)+\sum_{j=t k+r}^{\infty}(j+1-t k-\tau) a_{j} f\left(z_{j}\right)= \\
=C_{r+2}-C_{r+1}+\sum_{j=t k+r}^{\infty} a_{j} f\left(z_{j}\right),
\end{gathered}
$$

and consequently

$$
\begin{gathered}
\Delta^{2} z_{t k+r}=\Delta z_{t k+++1}-\Delta z_{t k+r}= \\
=C_{r+3}-2 C_{r+2}+C_{r+1}+\sum_{j=t k+r+1}^{\infty} a_{j} f\left(z_{j}\right)-\sum_{j=t k+r}^{\infty} a_{j} f\left(z_{j}\right)= \\
=C_{r+3}-2 C_{r+2}+C_{r+1}-a_{t k+r} f\left(z_{t k+r}\right), \\
r=0,1, \cdots, t-1, k>\frac{1}{t}\left(n_{1}+1-t\right)
\end{gathered}
$$

where

$$
C_{t+1}=C_{2}, C_{t+2}=C_{2}
$$

Denoting

$$
q_{t \hbar+r}=C_{r+3}-2 C_{r+2}+C_{r+1}, r=0,1, \cdots, t-1,
$$

we obtain the equation

$$
\triangle^{2} x_{n}+a_{n} f\left(x_{n}\right)=q_{n}
$$

which has an asymptotically $t$-periodic solution defined for $n>n_{1}$. This follows from (8) and $z_{t k+r} \in I_{t k+r}$, i.e. $z_{t k+r} \longrightarrow C_{r+1}$ as $k \longrightarrow \infty$.

It suffices to show that there exist a solution of (15) which coincides with (13) for $n>n_{1}$.

For this we observe that the equation (15) can be rewritten in equivalent form

$$
x_{n}+a_{n} f\left(x_{n}\right)=q_{n}-x_{n+2}+2 x_{n+1} .
$$


Taking $n=n_{1}, x_{n+1}=z_{n_{1}+1}, x_{n+2}=z_{n_{1}+2}$ we find $x_{n_{1}}$, which by the assumptions exists (probably more than one). Repeating this reasoning we find $x_{i}$ for $i=0,1, \cdots, n_{1}-1$. This function $x$ is of course a solution of (15) which coincides with $z$ for $n>n_{1}$ and therefore has the desired asymptotic behaviour.

Remark 3. If the functions $i_{R}+a_{n} f$ are one-to-one mappings of $\mathrm{R}$ onto $R$ then the solution obtained in the Theorem 3 is unique. The case $t=1$, i.e. the solutions having the asymptotic property $\lim _{n \rightarrow \infty} x_{n}=C$, was considered in the paper [1].

Let us observe that by Theorem 3 if we want to have some solutions which have a given asymptotically $t$-periodic solution, then it suffices to add to equation (E) the periodic perturbation $q$ which can be easily found by (14).

Example. As an example we consider the difference equation of the form

$$
\triangle^{2} x_{n}+\frac{(-1)^{n+1}}{4\left[2^{n}+(-1)^{n}\right]} x_{n}=0, n=1,2, \cdots
$$

It is evident by d'Alembert criterion that the series

$$
\sum_{j=1}^{\infty} \frac{j(-1)^{j+1}}{4\left[2^{j}+(-1)^{j}\right]}
$$

is absolutely convergent. Furthermore the functions

$$
x+\frac{(-1)^{n+1}}{4\left[2^{n}+(-1)^{n}\right]} x
$$

are surjections from $\mathbf{R}$ onto $R$ for all $n$. Therefore the assumptions of the Theorem 3 hold. We show that this equation has a $p_{2}^{\infty}$-function and find a 2-periodic solution of the form

$$
x_{n}=(-1)^{n}+y_{n} .
$$

Applying the proof of the Theorem 3 we see that the $p_{2}^{\infty}$-function $q$ takes the form

$$
q_{n}=4(-1)^{n}
$$

Considering the equation

$$
\triangle^{2} x_{n}+\frac{(-1)^{n+1}}{4\left[2^{n}+(-1)^{n}\right]} x_{n}=4(-1)^{n}
$$

we can observe that this equation has the solution

$$
x_{n}=(-1)^{n}+\frac{1}{2^{n}}
$$

which is of the desired form. 


\section{References}

1. A. DrozDowicz, J. POPENDA., Asymptotic Bahaviour of the Solutions of the Second Order Difference Equation, Proc. Amer. Math. Soc. (in press).

2. R. MusiblaK, On the Periodical Solutions of the System Linear Difference Equations, Fasc. Math 15 (1985), 141-150.

3. R. MUSIELAK, The Conditions for Existence of Periodical Solutions of the Second Order Difference Equation, Fasc.Math 15 (1985), 127-139.

R. Musielak: Institute of Mechanical Engineering

Technical University Poznań

ul. Piotrowo 3, 60-965 Poznań, POLAND.

J. Popenda: Institute of Mathematics

Technical University Ponnań

ul. Piotrowo 3, 60-965 Poznań, POLAND.

Rebut el 10 d'Octubre de 1986 